\title{
A blow-up dichotomy for semilinear fractional heat equations
}

\author{
Robert Laister $^{1}$ (D) Mikołaj Sierżęga ${ }^{2}$
}

Received: 18 December 2019 / Revised: 14 August 2020 / Published online: 23 September 2020

(c) The Author(s) 2020

\begin{abstract}
We derive a blow-up dichotomy for positive solutions of fractional semilinear heat equations on the whole space. That is, within a certain class of convex source terms, we establish a necessary and sufficient condition on the source for all positive solutions to become unbounded in finite time. Moreover, we show that this condition is equivalent to blow-up of all positive solutions of a closely-related scalar ordinary differential equation.
\end{abstract}

Mathematics Subject Classification 35A01 $\cdot 35 \mathrm{~B} 44 \cdot 35 \mathrm{~K} 58 \cdot 35 \mathrm{R} 11$

\section{Introduction}

In this paper we investigate the local and global existence properties of positive solutions of fractional semilinear heat equations of the form

$$
u_{t}=\Delta_{\alpha} u+f(u), \quad u(0)=\phi \in L^{\infty}\left(\mathbb{R}^{n}\right)
$$

where $\Delta_{\alpha}=-(-\Delta)^{\alpha / 2}$ denotes the fractional Laplacian operator with $0<\alpha \leq 2$ and $f$ satisfies the monotonicity condition

Communicated by Y.Giga.

Robert Laister

Robert.Laister@uwe.ac.uk

Mikołaj Sierżęga

M.Sierzega@mimuw.edu.pl

1 Department of Engineering Design and Mathematics, University of the West of England, Bristol BS16 1QY, UK

2 Faculty of Mathematics, Informatics and Mechanics, University of Warsaw, Banacha 2, 02-097 Warsaw, Poland 
(M) $f:[0, \infty) \rightarrow[0, \infty)$ is locally Lipschitz continuous, non-decreasing and $f(0)=0$.

We present a new dichotomy result for convex nonlinearities $f$ satisfying the ODE blow-up criterion

$$
\text { (B) } \int_{1}^{\infty} \frac{1}{f(u)} \mathrm{d} u<\infty \text {, }
$$

together with an additional, technical assumption (S) (see Sect. 2). Specifically, for this class of nonlinearities, we show that all positive solutions of (1.1) blow-up in $L^{\infty}\left(\mathbb{R}^{n}\right)$ in finite time if and only if

$$
\int_{0^{+}} \frac{f(u)}{u^{2+\alpha / n}} \mathrm{~d} u=\infty .
$$

Furthermore, we establish an equivalence between finite time blow-up of all positive solutions of (1.1) and finite time blow-up of all positive solutions of the scalar, nonautonomous ODE

$$
x^{\prime}=f(x)-\left(\frac{n}{\alpha t}\right) x, \quad x\left(t_{0}\right)=x_{0}>0 .
$$

To the best of our knowledge this kind of blow-up equivalence, between the PDE (1.1) and a scalar ODE such as (1.3), has not been established before.

We will refer to the phenomenon of blow-up in finite time of all non-negative, nontrivial solutions of (1.1) simply as the 'blow-up property'. We will also identify the phrase 'non-negative, non-trivial solution' synonymously with 'positive solution'.

For the case of classical diffusion $(\alpha=2)$ it has long been known that for $f$ convex and sufficiently large initial data $\phi$, blow-up in (1.1) occurs; see [15, Theorem 17.1] for bounded domains and the whole space alike. The central question then was whether diffusion could prevent blow-up for initial data sufficiently small. For general continuous sources $f$, this problem is highly non-trivial and remains open. However, under further restrictions on the form of the nonlinearity there has been significant progress, for example when $f$ is the power law nonlinearity $f(u)=u^{p}$. In [3] a threshold phenomenon was established, whereby the (Fujita) critical exponent, given by $p_{F}=1+2 / n$, separated two regimes: for $1<p<p_{F}$ (1.1) has the blow-up property, whereas for $p>p_{F}$ it is possible to find small initial conditions $\phi$ evolving into global-in-time solutions. Non-existence of positive global solutions in the delicate critical case $p=p_{F}$ was later established in [6] for the case $n \leq 2$ and subsequently by [17] for all $n \geq 1$. Thus was obtained the first blow-up dichotomy for (1.1): in the special case $f(u)=u^{p}$ and $\alpha=2$, (1.1) has the blow-up property if and only if $1<p \leq p_{F}$. Some slight generalisations can also be found in [4,5].

In fact the result obtained in [17, Theorem] extended previous work on blow-up in two important ways: firstly to convex sources terms $f$, and secondly to fractional diffusion operators. Specifically, it was shown for convex $f$ satisfying (M) and (B), that if

$$
\lim _{u \rightarrow 0} \frac{f(u)}{u^{p_{\alpha}}}>0, \quad \text { where } \quad p_{\alpha}:=1+\frac{\alpha}{n},
$$


then (1.1) has the blow-up property. In fact it is easy to see from the proofs in [17] that (1.4) need only hold in the limit inferior sense. There are many other works which consider the global and blow-up solution properties of nonlinear fractional diffusion equations, all assuming either a power law nonlinearity or a convex one bounded below by a power law near zero as in (1.4); see e.g., [2,7,8,12,14].

Subsequently it was shown in [9], in the special case of classical diffusion $(\alpha=2)$, that condition (1.4) is not necessary in order for (1.1) to have the blow-up property; this can be seen via the example in [9, Section 5] where a logarithmic-type correction of the critical Fujita case is considered. In that work it was shown ([9, Theorem 4.1]) that (1.1) has the blow-up property if $f$ is continuous on $[0, \infty)$, positive on $(0, \infty)$, $f(0)=0,(\mathbf{B})$ and (1.2) hold and $f$ satisfies a further technical condition (labelled (B.3) in [9]). In particular, neither monotonicity nor convexity of $f$ were required. On the other hand, this blow-up result is restricted to the case $\alpha=2$ and their technical condition (B.3) still imposes a certain logarithmic scaling bound near zero; see Sect. 4 later on for more details. Conversely, when (1.2) fails the authors in [9] go on to prove a global-in-time existence result for small initial data, subject to stronger regularity and monotonicity conditions on $f$.

An important aspect of this paper is that we demonstrate (via an explicit construction) that, for all $\alpha \in(0,2]$, there exist monotone, convex $f$ for which (1.1) has the blow-up property, but for which the results in [17] and [9] do not apply.

The remainder of the paper is organised as follows. In Sect. 2 we prove that, for a suitable class of sources $f,(1.2)$ is sufficient for the ODE (1.3) to have the blowup property. In Sect. 3 we show for this class that if the ODE (1.3) possesses the blow-up property then so too does (1.1). In Sect. 4 we present a construction which demonstrates that our assumption (S) (stated below) is strictly weaker than (B.3) of [9] in the case $\alpha=2$. We then establish in Sect. 5 the necessity of (1.2) for (1.1) to have the blow-up property and conclude with some remarks in Sect. 6.

\section{Blow-up of a related ODE}

Here we consider the blow-up properties of the non-autonomous ODE

$$
x^{\prime}=f(x)-\left(\frac{n}{\alpha t}\right) x, \quad x\left(t_{0}\right)=x_{0}>0, \quad t_{0}>0 .
$$

Definition 2.1 Suppose $f$ satisfies (M). We say that the ODE (2.1) has the blow-up property if the solution of (2.1) blows-up in finite time for every $x_{0}>0$ and $t_{0}>0$.

We now introduce some further hypotheses:

(C) $f$ is convex on $(0, \infty)$;

(S) there exist $c_{0}, \mu_{0}>0$ and $g:\left(\mu_{0}, \infty\right) \rightarrow(0, \infty)$ such that $\int_{\mu_{0}}^{\infty} 1 / g(s) \mathrm{d} s<\infty$ and

$$
f(\lambda \mu) \geq g(\mu) f(\lambda) \text { for all } \mu \geq \mu_{0} \text { and } \lambda \mu \in\left(0, c_{0}\right)
$$




\section{Remark 2.1}

(i) If $f(u) / u^{p}$ is non-decreasing near zero, on $\left(0, c_{0}\right)$ say, for some $p>1$, then (S) holds with $g(\mu)=\mu^{p}$. To see this, observe that for any choice of $\mu_{0} \geq 1$ we have, for $\mu \geq \mu_{0}$ and $\lambda \mu \in\left(0, c_{0}\right)$,

$$
\frac{f(\lambda \mu)}{(\lambda \mu)^{p}} \geq \frac{f(\lambda)}{\lambda^{p}}
$$

Hence $f(\lambda \mu) \geq \mu^{p} f(\lambda)$ for all $\mu \geq \mu_{0}$ and $\lambda \mu \in\left(0, c_{0}\right)$.

The particular, homogeneous, Fujita-critical case where $g(\mu)=\mu^{p}$ and $p=p_{F}$ was considered in $[9,(B .3)]$ on a strictly larger $\lambda-\mu$ region than appears in (S); i.e., the condition imposed upon $f$ in [9] is a more restrictive one than that in (S). We mention also that a condition such as $f(u) / u^{p}$ being non-decreasing was used in [1], although there the condition at infinity was relevant rather than near zero.

(ii) It is easy to verify that if $0 \neq f \in C^{1}$ satisfies (M) and (C) and the condition

$$
\liminf _{u \rightarrow 0} \frac{u f^{\prime}(u)}{f(u)}>1,
$$

then there exists a $p>1$ such that $f(u) / u^{p}$ is non-decreasing near zero. Consequently $f$ satisfies (S) by (i) above. Note that for $0 \neq f \in C^{1}$ satisfying (M) and (C), we always have $u f^{\prime}(u) / f(u) \geq 1$ for all $u>0$.

Theorem 2.1 Suppose $f$ satisfies $(\mathbf{M}),(\mathbf{C}),(\mathbf{B})$ and $(\mathbf{S})$. If

$$
\int_{0^{+}} \frac{f(u)}{u^{2+\alpha / n}} \mathrm{~d} u=\infty
$$

then the ODE (2.1) has the blow-up property.

Proof Suppose, for contradiction, that there exists a global solution $x(t)$ of (2.1). By ODE uniqueness it is clear that the solution of (2.1) is positive for all $t \geq t_{0}$. By (M) and (2.3), $f>0$ on $(0, \infty)$ and by $(\mathbf{C}), L(u):=f(u) / u$ is non-decreasing and positive for $u>0$.

Suppose first that $x$ is bounded away from zero, i.e., there exists $\varepsilon>0$ such that $x(t) \geq \varepsilon$ for all $t \geq t_{0}$. By monotonicity of $L, L(x(t)) \geq L(\varepsilon)>0$ for all $t \geq t_{0}$. Hence there exists $t_{1}>t_{0}$ such that

$$
1-\left(\frac{n}{\alpha t}\right) \frac{1}{L(x(t))} \geq 1-\frac{n}{\alpha L(\varepsilon) t} \geq 1 / 2
$$

for all $t \geq t_{1}$. For such $t$ we have

$$
x^{\prime}=f(x)\left(1-\left(\frac{n}{\alpha t}\right) \frac{1}{L(x(t))}\right) \geq f(x) / 2,
$$


and so by (B) $x$ blows up in finite time, a contradiction.

Now suppose that $x$ does not remain bounded away from zero. We then claim that $x^{\prime}(t) \leq 0$ for all $t \geq t_{0}$. For suppose this is not the case, so that there exists $t_{2} \geq t_{0}$ such that $x^{\prime}\left(t_{2}\right)>0$. Since $x$ is $C^{1}$ and not bounded away from zero, there exists $t_{3}>t_{2}$ such that $x^{\prime}(t)>0$ for all $t \in\left[t_{2}, t_{3}\right)$ and $x^{\prime}\left(t_{3}\right)=0$. Clearly $x\left(t_{2}\right)<x\left(t_{3}\right)$ and so by the monotonicity of $L$ we have

$$
0=\frac{x^{\prime}\left(t_{3}\right)}{x\left(t_{3}\right)}=L\left(x\left(t_{3}\right)\right)-\frac{n}{\alpha t_{3}}>L\left(x\left(t_{2}\right)\right)-\frac{n}{\alpha t_{2}}=\frac{x^{\prime}\left(t_{2}\right)}{x\left(t_{2}\right)}>0,
$$

which is clearly false. Hence $x^{\prime}(t) \leq 0$ for all $t \geq t_{0}$ as claimed. It follows that $x(t)$ is non-increasing and $x(t) \rightarrow 0$ as $t \rightarrow \infty$.

Now set $y(t)=t^{n / \alpha} x(t)$ so that $y$ satisfies the ODE

$$
y^{\prime}=y L\left(y t^{-n / \alpha}\right), \quad y\left(t_{0}\right)=t_{0}^{n / \alpha} x_{0}=: y_{0}>0 .
$$

By (2.4), $y$ is clearly increasing and so

$$
\begin{aligned}
y(t) & =\exp \left(\int_{t_{0}}^{t} L\left(y(s) s^{-n / \alpha}\right) \mathrm{d} s\right) \geq \exp \left(\int_{t_{0}}^{t} L\left(y_{0} s^{-n / \alpha}\right) \mathrm{d} s\right) \\
& =\exp \left(\frac{\alpha y_{0}^{\alpha / n}}{n} \int_{y_{0} t^{-n / \alpha}}^{x_{0}} \frac{f(u)}{u^{2+\alpha / n}} \mathrm{~d} u\right) \rightarrow \infty
\end{aligned}
$$

as $t \rightarrow \infty$, by (2.3). For $\tau>t_{0}$ sufficiently large we can ensure that $y(t) \geq \mu_{0}$ and $t^{-n / \alpha} y(t)=x(t) \leq c_{0}$ for all $t \geq \tau$. For such $t$ it follows from (S) that

$$
y^{\prime}=t^{n / \alpha} f\left(t^{-n / \alpha} y\right) \geq t^{n / \alpha} f\left(t^{-n / \alpha}\right) g(y)
$$

and so

$$
\int_{y(\tau)}^{y(t)} \frac{\mathrm{d} y}{g(y)} \geq \int_{\tau}^{t} L\left(s^{-n / \alpha}\right) \mathrm{d} s=\frac{\alpha}{n} \int_{t^{-n / \alpha}}^{\tau^{-n / \alpha}} \frac{f(u)}{u^{2+\alpha / n}} \mathrm{~d} u .
$$

Letting $t \rightarrow \infty$ and using (2.3) we again obtain a contradiction, on recalling the integrability of $1 / g$ in $(\mathbf{S})$.

\section{Blow-up of the PDE}

In this section we show that blow-up of the ODE (2.1) implies blow-up of the PDE (1.1). We denote by $\left\{S_{\alpha}(t)\right\}_{t \geq 0}$ the fractional heat semigroup on $L^{q}\left(\mathbb{R}^{n}\right)(q \geq 1)$ generated by $-\Delta_{\alpha}$ on $\mathbb{R}^{n}$ with the explicit representation formula

$$
\left[S_{\alpha}(t) \phi\right](x)=\int_{\mathbb{R}^{n}} K_{\alpha}(x-y, t) \phi(y) \mathrm{d} y, \quad \phi \in L^{q}\left(\mathbb{R}^{n}\right),
$$


where $K_{\alpha}$ is the (positive) fractional heat kernel. As is commonplace in the study of semilinear problems, we may then study (1.1) via the variation of constants formula

$$
u(t)=\mathscr{F}(u ; \phi):=S_{\alpha}(t) \phi+\int_{0}^{t} S_{\alpha}(t-s) f(u(s)) \mathrm{d} s .
$$

It is well known that for any non-negative initial condition $\phi \in L^{\infty}\left(\mathbb{R}^{n}\right)$ there is a $T_{\phi}>0$ such that (1.1) has a unique non-negative solution $u$ which is bounded on $\mathbb{R}^{n} \times[0, T]$ for any $T \in\left(0, T_{\phi}\right)$, such that if $T_{\phi}<\infty$ then $\|u(t)\|_{\infty} \rightarrow \infty$ as $t \rightarrow T_{\phi}$. If $T_{\phi}=\infty$ then we say that $u$ is a global solution of (1.1).

Definition 3.1 Suppose $f$ satisfies (M). We say that the PDE (1.1) has the blow-up property if for every non-trivial, non-negative $\phi \in L^{\infty}\left(\mathbb{R}^{n}\right)$ we have $T_{\phi}<\infty$.

Theorem 3.1 Suppose that $f$ satisfies $(\mathbf{M})$ and $(\mathbf{C})$. If the ODE (2.1) has the blow-up property then the PDE (1.1) has the blow-up property.

Proof We proceed as in the proof of the main theorem in [17, Section 4]. We briefly outline the initial steps of that proof for the reader's convenience.

Suppose, for contradiction, that $u$ is a non-negative, global solution of (1.1). Then $u$ satisfies the integral equation

$$
u(x, t)=\int_{\mathbb{R}^{n}} K_{\alpha}(x-y, t) \phi(y) \mathrm{d} y+\int_{0}^{t} \int_{\mathbb{R}^{n}} K_{\alpha}(x-y, t-s) f(u(y, s)) \mathrm{d} y \mathrm{~d} s .
$$

Clearly $u>0$ for all $t>0$ and so, by translating in time if necessary, we may assume without loss of generality that $\phi>0$.

Using the integral formulation (3.3), positivity of the solution and standard properties of $K_{\alpha}$, one can then show that there exist constants $c>0, \tau_{0}>0$ and $t_{0}>0$ such that $u\left(x, t_{0}\right) \geq c K_{\alpha}\left(x, \tau_{0}\right)$ for all $x \in \mathbb{R}^{n}$ (see [17, p. 48]). It follows that

$$
\begin{aligned}
u\left(x, t+t_{0}\right)= & \int_{\mathbb{R}^{n}} K_{\alpha}(x-y, t) u\left(y, t_{0}\right) \mathrm{d} y \\
& +\int_{0}^{t} \int_{\mathbb{R}^{n}} K_{\alpha}(x-y, t-s) f\left(u\left(y, s+t_{0}\right)\right) \mathrm{d} y \mathrm{~d} s \\
\geq & c K_{\alpha}\left(x, t+\tau_{0}\right)+\int_{0}^{t} \int_{\mathbb{R}^{n}} K_{\alpha}(x-y, t-s) f\left(u\left(y, s+t_{0}\right)\right) \mathrm{d} y \mathrm{~d} s .
\end{aligned}
$$

Setting $v(x, t)=u\left(x, t+t_{0}\right)$ yields

$$
v(x, t)=c K_{\alpha}\left(x, t+\tau_{0}\right)+\int_{t_{0}}^{t} \int_{\mathbb{R}^{n}} K_{\alpha}(x-y, t-s) f(v(y, s)) \mathrm{d} y \mathrm{~d} s .
$$

Clearly $v(t) \in L^{\infty}\left(\mathbb{R}^{n}\right)$ for all $t>0$ since $u$ is assumed to be in $L^{\infty}\left(\mathbb{R}^{n}\right)$ for all $t>0$. Now set

$$
z(t)=\int_{\mathbb{R}^{n}} K_{\alpha}(x, t) v(x, t) \mathrm{d} x
$$


Evidently $z(t)$ is positive and finite for all $t>0$. Multiplying (3.4) by $K_{\alpha}(x, t)$, integrating over $\mathbb{R}^{n}$ and using the semigroup property of $K_{\alpha}$, gives

$$
z(t)=k\left(2 t+\tau_{0}\right)^{-n / \alpha}+\int_{t_{0}}^{t} \int_{\mathbb{R}^{n}} K_{\alpha}(y, 2 t-s) f(v(y, s)) \mathrm{d} y \mathrm{~d} s .
$$

where $k=k(n, \alpha, c)$ is a positive constant. Now using the scaling property

$$
K_{\alpha}(x, t)=t^{-n / \alpha} K_{\alpha}\left(t^{-1 / \alpha} x, 1\right)
$$

of $K_{\alpha}$ (see e.g., [17, p.46-47]) and the fact that $K_{\alpha}(x, t)$ is decreasing in $|x|$, we have for $s \leq t$,

$$
\begin{aligned}
K_{\alpha}(y, 2 t-s) & =(2 t-s)^{-n / \alpha} K_{\alpha}\left((2 t-s)^{-1 / \alpha} y, 1\right) \\
& \geq(2 t-s)^{-n / \alpha} K_{\alpha}\left(s^{-1 / \alpha} y, 1\right) \\
& =\left(\frac{2 t-s}{s}\right)^{-n / \alpha} K_{\alpha}(y, s) \\
& \geq 2^{-n / \alpha}(t / s)^{-n / \alpha} K_{\alpha}(y, s) .
\end{aligned}
$$

Hence, by Jensen's inequality,

$$
\begin{aligned}
z(t) & \geq k\left(2 t+\tau_{0}\right)^{-n / \alpha}+2^{-n / \alpha} \int_{t_{0}}^{t}(t / s)^{-n / \alpha} \int_{\mathbb{R}^{n}} K_{\alpha}(y, s) f(v(y, s)) \mathrm{d} y \mathrm{~d} s \\
& \geq k\left(2 t+\tau_{0}\right)^{-n / \alpha}+2^{-n / \alpha} \int_{t_{0}}^{t}(t / s)^{-n / \alpha} f(z(s)) \mathrm{d} s \\
& \geq \kappa t^{-n / \alpha}+\kappa \int_{t_{0}}^{t}(t / s)^{-n / \alpha} f(z(s)) \mathrm{d} s
\end{aligned}
$$

for all $t \geq t_{1}, \kappa<2^{-n / \alpha} \min \{1, k\}$ and $t_{1}>t_{0}$ sufficiently large. Here we point out that (3.5) is a departure from the form used in [17, Equation (4.4)] and is the reason for our introduction and analysis of the auxiliary ODE (2.1).

It now follows from (3.5) that for $t>t_{1}, z$ is a supersolution of the ODE

$$
w^{\prime}=\kappa f(w)-\left(\frac{n}{\alpha t}\right) w
$$

By rescaling time $(t \mapsto \kappa t)$ we see that $z(t) \geq x(\kappa t)$, where $x$ is the solution of the ODE

$$
x^{\prime}=f(x)-\left(\frac{n}{\alpha t}\right) x, \quad x\left(\kappa t_{1}\right)=z\left(t_{1}\right)>0 .
$$

By assumption $x$ (and hence $z$ ) blows up in finite time, yielding the required contradiction to our earlier statement that $z(t)$ is finite for all $t>0$. 
By Theorems 2.1 and 3.1 we obtain the following blow-up result for (1.1).

Corollary 3.2 Suppose $f$ satisfies $(\mathbf{M}),(\mathbf{C}),(\mathbf{B})$ and $(\mathbf{S})$. If

$$
\int_{0^{+}} \frac{f(u)}{u^{2+\alpha / n}} \mathrm{~d} u=\infty,
$$

then the PDE (1.1) has the blow-up property.

\section{A distinguishing example}

In this section we present an example of a function $f$ which satisfies the hypotheses of Corollary 3.2, but not those of [9, Theorem 4.1] (for $\alpha=2$ ) nor [17, Theorem]. Firstly, the $f$ we construct below has the property

$$
\liminf _{u \rightarrow 0} \frac{f(u)}{u^{p_{\alpha}}}=0
$$

so that the requirement (1.4) (labelled (F.2) in [17]) does not hold.

In [9, Theorem 4.1] the authors use a similar but more restrictive version of (S) (there denoted by assumption (B3)) to establish blow-up of the PDE (1.1) when $\alpha=2$. The authors assume that there exists $c_{0} \in(0,1]$ such that

$$
f(\lambda \mu) \geq c_{0} \mu^{p_{F}} f(\lambda) \text { for all } 0<\lambda \leq \mu, \quad \lambda \in\left(0, c_{0}\right) \text { and } \lambda \mu \in\left(0, c_{0}\right) .
$$

Hence the rôle of $g$ in $(\mathbf{S})$ is played there by the power law nonlinearity $g(\mu)=\mu^{p_{F}}$ (recall Remark 2.1). In fact the homogeneity of this power law function is crucial in the proof of [9, Theorem 2.1] (see also [9, Theorem 3.5]) on which [9, Theorem 4.1] relies. Indeed, the iterative blow-up procedure used in the proof of [9, Theorem 2.1] utilizes in an essential way certain scaling identities relating the exponential function in the Gaussian heat kernel $K_{2}$ and the power law. Furthermore, (S) is not required to hold for arbitrarily small $\mu$ which is an essential requirement in the proof of [9, Theorem 2.1]. On the other hand we impose the stronger convexity assumption in (C), absent in [9].

Now observe, upon taking $\lambda=\mu$, that any $f$ satisfying (4.2) necessarily satisfies

$$
\liminf _{\lambda \rightarrow 0} \frac{f\left(\lambda^{2}\right)}{\lambda^{p_{F}} f(\lambda)}>0 .
$$

(Note that this in turn imposes upon $f$ a kind of logarithmic scaling bound, as emerges in the proof of [9, Lemma 3.6]). The $f$ we construct below will satisfy the hypotheses of Corollary 3.2 (for any $\alpha \in(0,2]$ ), but not (4.3) (for $\alpha=2$ ).

Let $\alpha \in(0,2]$.

Step 1. Define the monotonically decreasing sequences

$$
\sigma_{i}:=e^{-i^{2}}, \quad u_{i}:=e^{-e^{i^{2}}}, \quad i \in \mathbb{N} \text {. }
$$


Let $I_{i}$ denote the interval $I_{i}=\left[u_{i+1}, u_{i}\right)$ and define

$$
\tilde{f}(u)=\sigma_{i} u^{p_{\alpha}}, \quad u \in I_{i}, \quad i \in \mathbb{N}
$$

with $\tilde{f}(0)=0$. Notice that $\tilde{f}(u)=u^{p_{\alpha}} / \ln \left(1 / u_{i}\right)$ on $I_{i}$, so that $\tilde{f}$ models a logarithmic correction to the critical power law case on a sequence of vanishingly small intervals near zero, to be compared with the example of [9, Section 5]. It is clear that $\tilde{f}$ is non-decreasing on $[0, \delta]$ (where $\delta>0$ can be chosen as small as desired later on) with discontinuities at $u=u_{i}$.

Step 2 . We now modify $\tilde{f}$ to create a function $f$ satisfying $(\mathbf{M}),(\mathbf{C})$ and $(\mathbf{B})$.

Fix $p$ and $\theta>1$ such that

$$
1<p<p_{\alpha}, \quad \frac{p_{\alpha}}{p_{\alpha}-1}<\theta<\frac{p}{p-1}
$$

and set $v_{i}=\theta u_{i+1}$. It is easily verified that $\theta u_{i+1}<u_{i}$ for all $i$ sufficiently large, and so for all such $i$

$$
u_{i+1}<v_{i}<u_{i}
$$

Now set $J_{i}=\left[u_{i+1}, v_{i}\right)$ and $M_{i}=\left[v_{i}, u_{i}\right)$ (so that $I_{i}$ is the disjoint union of $J_{i}$ and $\left.M_{i}\right)$ and define

$$
f(u)= \begin{cases}b_{i} u-a_{i}, & u \in J_{i}, \\ \sigma_{i} u^{p_{\alpha}}, & u \in M_{i}\end{cases}
$$

for $i$ large, with $f(0)=0$. Note that $f\left(v_{i}\right)=\sigma_{i} v_{i}^{p_{\alpha}}$ so that (4.1) holds.

We now choose $a_{i}$ and $b_{i}$ to ensure that $f$ is continuous, i.e. such that the line $y=b_{i} u-a_{i}$ passes through the points $\left(u_{i+1}, \sigma_{i+1} u_{i+1}^{p_{\alpha}}\right)$ and $\left(v_{i}, \sigma_{i} v_{i}^{p_{\alpha}}\right)=$ $\left(\theta u_{i+1}, \sigma_{i} \theta^{p_{\alpha}} u_{i+1}^{p_{\alpha}}\right)$. This yields

$$
b_{i}=\frac{u_{i+1}^{p_{\alpha}-1}\left(\theta^{p_{\alpha}} \sigma_{i}-\sigma_{i+1}\right)}{\theta-1}>0, \quad a_{i}=\frac{u_{i+1}^{p_{\alpha}}\left(\theta^{p_{\alpha}} \sigma_{i}-\theta \sigma_{i+1}\right)}{\theta-1}>0 .
$$

By construction $f$ is also increasing and Lipschitz on $[0, \delta]$. In order that $f$ be convex on $[0, \delta]$ we require that

$$
p_{\alpha} \sigma_{i+1} u_{i+1}^{p_{\alpha}-1} \leq b_{i} \leq p_{\alpha} \sigma_{i} v_{i}^{p_{\alpha}-1}
$$

(by comparing the gradient of $f$ at the endpoints of the intervals), or equivalently

$$
\theta^{p_{\alpha}-1}\left(\theta-p_{\alpha}(\theta-1)\right) \leq \frac{\sigma_{i+1}}{\sigma_{i}} \leq \frac{\theta^{p_{\alpha}}}{1+p_{\alpha}\left(\theta^{p_{\alpha}}-1\right)}
$$

By (4.5), and since $\sigma_{i+1} / \sigma_{i} \rightarrow 0$ as $i \rightarrow \infty$, (4.7) holds for all $i$ sufficiently large. 
Thus, $f$ is increasing, convex and Lipschitz on $[0, \delta]$. It is clear that the domain of $f$ can then be extended to $[0, \infty)$ while still preserving monotonicity, convexity and Lipschitz continuity and also such that (B) holds.

Step 3. Next we show that $f$ satisfies the remaining hypotheses of Corollary 3.2. By Remark 2.1(i) it suffices to show that

(i) $f(u) / u^{p}$ is non-decreasing on $(0, \delta)$, and

(ii) $\int_{0^{+}} \frac{f(u)}{u^{2+\alpha / n}} \mathrm{~d} u=\infty$.

For (i) let $F(u):=f(u) / u^{p}$. This continuous, piecewise differentiable function is given explicitly by

$$
F(u)= \begin{cases}b_{i} u^{1-p}-a_{i} u^{-p}, & u \in J_{i}, \\ \sigma_{i} u^{p_{\alpha}-p}, & u \in M_{i}\end{cases}
$$

Clearly $F$ is non-decreasing on $M_{i}$ for all $i$. On $J_{i}=\left[u_{i+1}, v_{i}\right)$ we have that

$$
F^{\prime}(u)=u^{-p-1}\left(p a_{i}-(p-1) b_{i} u\right)
$$

Hence $F^{\prime} \geq 0$ on $J_{i}$ if and only if

$$
p a_{i} \geq(p-1) b_{i} v_{i}
$$

Now, recalling (4.6), we have

$$
\begin{aligned}
\frac{p a_{i}}{(p-1) b_{i} v_{i}} & =\frac{p\left(\theta^{p_{\alpha}} \sigma_{i}-\theta \sigma_{i+1}\right)}{\theta(p-1)\left(\theta^{p_{\alpha}} \sigma_{i}-\sigma_{i+1}\right)} \\
& =\frac{p\left(\theta^{p_{\alpha}}-\theta \sigma_{i+1} / \sigma_{i}\right)}{\theta(p-1)\left(\theta^{p_{\alpha}}-\sigma_{i+1} / \sigma_{i}\right)} \rightarrow \frac{p}{\theta(p-1)}
\end{aligned}
$$

as $i \rightarrow \infty$. Hence, by (4.5), (4.8) holds for all $i$ sufficiently large. Thus $F$ is nondecreasing on $(0, \delta)$.

For (ii),

$$
\begin{aligned}
\int_{0}^{\delta} \frac{f(u)}{u^{2+\alpha / n}} \mathrm{~d} u & \geq \sum_{i=1}^{\infty} \int_{M_{i}} \frac{f(u)}{u^{2+\alpha / n}} \mathrm{~d} u=\sum_{i=1}^{\infty} \int_{v_{i}}^{u_{i}} \frac{\sigma_{i}}{u} \mathrm{~d} u \\
& =\sum_{i=1}^{\infty} \sigma_{i} \log \left(u_{i} / v_{i}\right)=\sum_{i=1}^{\infty} \sigma_{i} \log \left(u_{i} /\left(\theta u_{i+1}\right)\right) \\
& =\sum_{i=1}^{\infty}\left(e^{2 i+1}-1\right)-\log \theta \sum_{i=1}^{\infty} e^{-i^{2}} \\
& =\infty
\end{aligned}
$$

recalling (4.4). 
Step 4. Finally we show that $f$ fails to satisfy assumption $[9,(\mathrm{~B} 3)]$ when $\alpha=2$. In fact we establish a more general result: for any $\alpha \in(0,2]$, we find a sequence $\lambda_{i} \rightarrow 0$ such that

$$
\lim _{i \rightarrow \infty} \frac{f\left(\lambda_{i}^{2}\right)}{\lambda_{i}^{p_{\alpha}} f\left(\lambda_{i}\right)}=0
$$

Consequently (4.3) fails in the special case $\alpha=2$.

To achieve this we show that there is a sequence $\lambda_{i} \in M_{i}=\left[v_{i}, u_{i}\right)$ such that $\lambda_{i}^{2} \in M_{i+1}$. It will then follow that

$$
\lim _{i \rightarrow \infty} \frac{f\left(\lambda_{i}^{2}\right)}{\lambda_{i}^{p_{\alpha}} f\left(\lambda_{i}\right)}=\lim _{i \rightarrow \infty} \frac{\sigma_{i+1}\left(\lambda_{i}^{2}\right)^{p_{\alpha}}}{\lambda_{i}^{p_{\alpha}}\left(\sigma_{i} \lambda_{i}^{p_{\alpha}}\right)}=\lim _{i \rightarrow \infty} \frac{\sigma_{i+1}}{\sigma_{i}}=0
$$

recalling that $\sigma_{i}=e^{-i^{2}}$.

Fix $1 / 2<q<1$ and let $\lambda_{i}=v_{i}^{q}$. Clearly $\lambda_{i}>v_{i}$ since $v_{i}<1$ and $q<1$. It is also easily verified that $\lambda_{i}=\theta^{q} u_{i+1}^{q}<u_{i}$ for $i$ sufficiently large, recalling (4.4). Hence $\lambda_{i} \in M_{i}$ for such $i$. Next,

$$
\lambda_{i}^{2}=v_{i}^{2 q}=\theta^{2 q} u_{i+1}^{2 q}<u_{i+1}
$$

for $i$ sufficiently large, since $2 q>1$. Also, $\lambda_{i}^{2}=v_{i}^{2 q}>u_{i+1}^{2 q}$ and $v_{i+1}=\theta u_{i+2}$. Hence in order to show that $\lambda_{i}^{2}>v_{i+1}$, it suffices to show that $u_{i+1}^{2 q}>\theta u_{i+2}$. This is readily verified for large $i$, recalling (4.4). It follows that $\lambda_{i}^{2} \in M_{i+1}$, as required.

Remark 4.1 Consider the case $\alpha=2$. By taking $g(\mu)=\mu^{p_{F}}$, any function $f$ satisfying (4.2) necessarily satisfies our condition (S). Hence any convex function $f$ satisfying the hypotheses of [9, Theorem 4.1] also satisfies those of Corollary 3.2. Our distinguishing example therefore shows that, within the class of convex source terms, Corollary 3.2 is strictly stronger than [9, Theorem 4.1].

Remark 4.2 It is reasonable to speculate whether the analogous condition to (4.2), with the power law $\mu^{p_{F}}$ replaced by $\mu^{p_{\alpha}}$, might provide the basis for similar results to those in [9] for the fractional diffusion case $0<\alpha<2$. However, the $f$ constructed above satisfies

$$
\liminf _{\lambda \rightarrow 0} \frac{f\left(\lambda^{2}\right)}{\lambda p_{\alpha} f(\lambda)}=0
$$

for any $\alpha \in(0,2]$. Consequently, the $f$ constructed above pre-empts any improvements that might possibly be obtained in this way, at least within the class of convex source terms. 


\section{Global existence}

In this section we consider the issue of global continuation of locally bounded solutions of (1.1). We set $Q_{T}=\mathbb{R}^{n} \times(0, T)$ and write $\|\cdot\|_{q}$ for the norm in $L^{q}\left(\mathbb{R}^{n}\right)$.

Definition 5.1 Let $T>0$. We say that a non-negative, measurable, finite almost everywhere function $w: Q_{T} \rightarrow \mathbb{R}$ is an integral supersolution of (1.1) on $Q_{T}$ if $w$ satisfies $\mathscr{F}(w ; \phi) \leq w$ almost everywhere in $Q_{T}$, with $\mathscr{F}$ as in (3.2).

We recall the following well-known smoothing estimate for the fractional heat semigroup for $1 \leq q \leq r \leq \infty$ and $\phi \in L^{q}\left(\mathbb{R}^{n}\right)$ (see e.g., [13, Lemma 3.1]):

$$
\left\|S_{\alpha}(t) \phi\right\|_{r} \leq C t^{-\frac{n}{\alpha}\left(\frac{1}{q}-\frac{1}{r}\right)}\|\phi\|_{q}, \quad t>0
$$

where $C=C(n, \alpha, q, r)$.

For $f$ satisfying $(\mathbf{M})$ we define the non-decreasing function $\ell:(0, \infty) \rightarrow[0, \infty)$ by

$$
\ell(u)=\sup _{0<s \leq u} \frac{f(s)}{s} .
$$

Theorem 5.1 Suppose $\phi \in L^{1}\left(\mathbb{R}^{n}\right) \cap L^{\infty}\left(\mathbb{R}^{n}\right), \phi \geq 0$ and $f$ satisfies $(\mathbf{M})$. Let $u(t ; \phi)$ denote the unique, non-negative solution of (1.1) with maximal interval of existence $\left[0, T_{\phi}\right)$. If

$$
\int_{0^{+}} u^{-p_{\alpha}} \ell(u) \mathrm{d} u<\infty
$$

then there exists $\rho>0$ such that for all $\phi$ satisfying $\|\phi\|_{1}+\|\phi\|_{\infty} \leq \rho$ we have $T_{\phi}=\infty$ and

$$
0 \leq u(t ; \phi) \leq 2 S_{\alpha}(t) \phi
$$

for all $t \geq 0$. Consequently $\|u(t ; \phi)\|_{\infty} \leq 2 C t^{-n / \alpha}\|\phi\|_{1}$ for all $t>0$, where $C=$ $C(n, \alpha, 1, \infty)$.

Proof We will show that for suitably small $\rho>0, w:=2 S_{\alpha}(t) \phi$ is an integral supersolution of (1.1) for all $t \geq 0$. Via the monotone iteration scheme $u_{n+1}=$ $\mathscr{F}\left(u_{n} ; \phi\right)$ we then obtain a decreasing sequence of functions $u_{n}$ such that $0 \leq u_{n} \leq w$ and converging to a solution $\tilde{u}(t ; \phi)$ of $(1.1)$. See, for example, $[10,16]$ for the case $\alpha=2$ and [11] for the fractional case for general results of this kind. By standard uniqueness results we may then deduce that $\tilde{u}(t ; \phi)=u(t ; \phi)$ and $0 \leq u(t ; \phi) \leq w$, yielding (5.4). The $L^{\infty}$-bound for $u$ then follows by $L^{1}-L^{\infty}$ smoothing. 
First set $C_{1}=C(n, \alpha, \infty, \infty)$ and choose $\rho$ such that $\rho C_{1} \leq 1$. Then choose $\tau>0$ such that $2 \ell(2) \tau<1$. By (5.1) we have $\left\|S_{\alpha}(t) \phi\right\|_{\infty} \leq C_{1}\|\phi\|_{\infty} \leq C_{1} \rho \leq 1$ for all $t>0$. In particular, for all $t \in(0, \tau]$ we have

$$
\begin{aligned}
\mathscr{F}(w ; \phi)-w & =S_{\alpha}(t) \phi+\int_{0}^{t} S_{\alpha}(t-s) f(w(s)) \mathrm{d} s-w \\
& \leq S_{\alpha}(t) \phi+\int_{0}^{t} S_{\alpha}(t-s)[\ell(w(s)) w(s)] \mathrm{d} s-w \\
& =\int_{0}^{t} S_{\alpha}(t-s)\left[\ell\left(2 S_{\alpha}(s) \phi\right) 2 S_{\alpha}(s) \phi\right] \mathrm{d} s-S_{\alpha}(t) \phi \\
& \leq \int_{0}^{t} S_{\alpha}(t-s)\left[\ell\left(\left\|2 S_{\alpha}(s) \phi\right\|_{\infty}\right) 2 S_{\alpha}(s) \phi\right] \mathrm{d} s-S_{\alpha}(t) \phi \\
& \leq \int_{0}^{t} S_{\alpha}(t-s)\left[2 \ell(2) S_{\alpha}(s) \phi\right] \mathrm{d} s-S_{\alpha}(t) \phi \\
& =2 \ell(2) \int_{0}^{t} S_{\alpha}(t) \phi \mathrm{d} s-S_{\alpha}(t) \phi \\
& \leq(2 \ell(2) \tau-1) S_{\alpha}(t) \phi \\
& \leq 0 .
\end{aligned}
$$

For $t>\tau$ we proceed as above, utilizing the $L^{1}-L^{\infty}$ smoothing estimate $\left\|S_{\alpha}(t) \phi\right\|_{\infty} \leq$ $C_{2} t^{-n / \alpha}\|\phi\|_{1} \leq C_{2} \rho t^{-n / \alpha}$, where $C_{2}:=C(n, \alpha, 1, \infty)$. Whence,

$$
\begin{aligned}
\mathscr{F}(w ; \phi)-w & =S_{\alpha}(t) \phi+\int_{0}^{\tau} S_{\alpha}(t-s) f(w(s)) \mathrm{d} s+\int_{\tau}^{t} S_{\alpha}(t-s) f(w(s)) \mathrm{d} s-w \\
& \leq(2 \ell(2) \tau-1) S_{\alpha}(t) \phi+\int_{\tau}^{t} S_{\alpha}(t-s)[\ell(w(s)) w(s)] \mathrm{d} s \\
& \leq(2 \ell(2) \tau-1) S_{\alpha}(t) \phi+\int_{\tau}^{t} S_{\alpha}(t-s)\left[\ell\left(2 S_{\alpha}(s) \phi\right) 2 S_{\alpha}(s) \phi\right] \mathrm{d} s \\
& \leq(2 \ell(2) \tau-1) S_{\alpha}(t) \phi+\int_{\tau}^{t} S_{\alpha}(t-s)\left[\ell\left(\left\|2 S_{\alpha}(s) \phi\right\|_{\infty}\right) 2 S_{\alpha}(s) \phi\right] \mathrm{d} s \\
& \leq(2 \ell(2) \tau-1) S_{\alpha}(t) \phi+\int_{\tau}^{t} S_{\alpha}(t-s)\left[\ell\left(2 C_{2} \rho s^{-n / \alpha}\right) 2 S_{\alpha}(s) \phi\right] \mathrm{d} s \\
& =(2 \ell(2) \tau-1) S_{\alpha}(t) \phi+2 S_{\alpha}(t) \phi \int_{\tau}^{t} \ell\left(2 C_{2} \rho s^{-n / \alpha}\right) \mathrm{d} s \\
& =\left(2 \ell(2) \tau-1+(\alpha / n) 2^{p_{\alpha}}\left(C_{2} \rho\right)^{\alpha / n} \int_{2 C_{2} \rho t^{-n / \alpha}}^{2 C_{2} \rho \tau^{-n / \alpha}} x^{-p_{\alpha}} \ell(x) \mathrm{d} x\right) S_{\alpha}(t) \phi \\
& \leq\left(2 \ell(2) \tau-1+(\alpha / n) 2^{p_{\alpha}}\left(C_{2} \rho\right)^{\alpha / n} \int_{0}^{2 C_{2} \rho \tau^{-n / \alpha}} x^{-p_{\alpha}} \ell(x) \mathrm{d} x\right) S_{\alpha}(t) \phi \\
& \leq 0
\end{aligned}
$$

for $\rho$ sufficiently small (and independently of $t$ ), by (5.3). 
We are now in a position to state our main result.

Theorem 5.2 (Blow-up Dichotomy) If $f$ satisfies $(\mathbf{M}),(\mathbf{C}),(\mathbf{B})$ and $(\mathbf{S})$, then the following are equivalent:

(a) the PDE (1.1) has the blow-up property;

(b) the ODE (2.1) has the blow-up property;

(c) $\int_{0^{+}} \frac{f(u)}{u^{2+\alpha / n}} \mathrm{~d} u=\infty$.

Proof By the contrapositive of Theorem 5.1, (a) implies (c) (noting that $\ell(u)=f(u) / u$ for $f$ convex). By Theorem 2.1, (c) implies (b). By Theorem 3.1, (b) implies (a).

Example 5.1 In the special case of the homogeneous power law nonlinearity $f(u)=$ $u^{p}$ with $p>1$, Theorem 5.2 shows that (1.1) has the blow-up property if and only if $1<p \leq p_{\alpha}$, as is well known [3,4,6,17].

Example 5.2 For $\beta>0$ take

$$
f(u)= \begin{cases}u^{p_{\alpha}}(\log (1 / u))^{-\beta}, & u \in\left(0, c_{0}\right), \\ \hat{f}(u), & u \geq c_{0},\end{cases}
$$

with $f(0)=0$ and $\hat{f}$ and $c_{0}>0$ to be specified. For sufficiently small $c_{0}>0, f$ can be shown to be convex (via a tedious calculation) and $f(u) / u^{p}$ is non-decreasing on $\left(0, c_{0}\right)$ for any $p \in\left(1, p_{\alpha}\right]$. By Remark 2.1 (i) it follows that $f$ satisfies (S). Clearly $\hat{f}$ can then be chosen such that $(\mathbf{M}),(\mathbf{B})$ and $(\mathbf{C})$ hold. Computation of the integral in Theorem 5.2 (c) then shows that (1.1) has the blow-up property if and only if $0<\beta \leq 1$. See the example in [9, Section 5] for the special case $\alpha=2$.

Remark 5.1 Under the assumptions of Theorem 5.2 we could rewrite the equivalences as follows:

(a) there exist positive, global solutions of the PDE (1.1);

(b) there exist positive, global solutions of the ODE (2.1);

(c) $\int_{0^{+}} \frac{f(u)}{u^{2+\alpha / n}} \mathrm{~d} u<\infty$.

\section{Concluding remarks}

We have established a new blow-up dichotomy for positive solutions of fractional semilinear heat equations, extending those of $[3,6,9,17]$. In particular, for a class of convex nonlinearities we have established an equivalence between the PDE (1.1) and the ODE (2.1) with respect to the blow-up property. Furthermore we have determined necessary and sufficient conditions on the nonlinearity $f$, in the form of a non-integrability condition near zero, for this blow-up property to hold.

When viewed in their integral form, there is an obvious formal similarity between the PDE and an auxiliary ODE: the PDE (1.1) can be cast as

$$
u(t)=S_{\alpha}(t) \phi+\int_{0}^{t} S_{\alpha}(t-s) f(u(s)) \mathrm{d} s,
$$


while the ODE (2.1) can be written as

$$
x(t)=\left(t / t_{0}\right)^{-n / \alpha} x\left(t_{0}\right)+\int_{t_{0}}^{t}(t / s)^{-n / \alpha} f(x(s)) \mathrm{d} s .
$$

The similarity arises when considering the decay rate of the operator norm of $S_{\alpha}(t)$ : $L^{1}\left(\mathbb{R}^{n}\right) \rightarrow L^{\infty}\left(\mathbb{R}^{n}\right)$, which is given (via the smoothing estimate (5.1)) by

$$
\left\|S_{\alpha}(t)\right\|_{L^{1} \rightarrow L^{\infty}} \leq C t^{-n / \alpha}
$$

It is intriguing that this formal similarity manifests itself as an equivalence with respect to the blow-up property.

It would be interesting to know whether the technical hypothesis (S) can be removed in Theorem 2.1 and consequently in Theorem 5.2. This would yield a sharper and perhaps more natural result. However, recalling Remark 2.1 (i), we suspect that the stronger (but more easily verified) assumption that $f(u) / u^{p}$ be non-decreasing near zero for some $p>1$, will prove more useful in applications. Similarly we would like to better understand the rôle of the convexity assumption on $f$. It is this convexity that permits us to show, via Jensen's inequality, that blow-up of the ODE implies blow-up of the PDE. It remains open whether our blow-up equivalence result can be obtained without the convexity assumption and without assuming (S).

Finally, we mention that the analysis of fractional semilinear parabolic equations such as (1.1) is intimately related to the study of symmetric $\alpha$-stable processes in probability theory. It seems reasonable to hope that our work might have parallels in that domain and provide new insights for such processes.

Acknowledgements RL was partially supported by the QJMAM Fund for Applied Mathematics. MS was partially supported by NCN grant 2017/26/D/ST1/00614.

Open Access This article is licensed under a Creative Commons Attribution 4.0 International License, which permits use, sharing, adaptation, distribution and reproduction in any medium or format, as long as you give appropriate credit to the original author(s) and the source, provide a link to the Creative Commons licence, and indicate if changes were made. The images or other third party material in this article are included in the article's Creative Commons licence, unless indicated otherwise in a credit line to the material. If material is not included in the article's Creative Commons licence and your intended use is not permitted by statutory regulation or exceeds the permitted use, you will need to obtain permission directly from the copyright holder. To view a copy of this licence, visit http://creativecommons.org/licenses/by/4.0/.

\section{References}

1. Baras, P., Cohen, L.: Complete blow-up after $T_{\max }$ for the solution of a semilinear heat equation. J. Funct. Anal. 71, 142-174 (1987)

2. Birkner, M., López-Mimbela, J.A., Wakolbinger, A.: Comparison results and steady states for the Fujita equation with fractional Laplacian. Ann. Inst. H. Poincaré Anal. Non Linéaire. 22, 83-97 (2005)

3. Fujita, H.: On the blowing up of solutions of the Cauchy problem for $u_{t}=\Delta u+u^{1+\alpha}$. J. Sci. Univ. Tokyo Sect. I 13, 109-124 (1966)

4. Fujita, H.: On some nonexistence and nonuniqueness theorems for nonlinear parabolic equations. In: Browder, F.E. (ed.) Proc. Symp. Pure Math., vol. XVIII, pp. 105-113. Nonlinear Functional Analysis, Part 1. AMS, Providence (1970) 
5. Fujita, H., Watanabe, S.: On the uniqueness and non-uniqueness of solutions of initial value problems for some quasi-linear parabolic equations. Commun. Pure Appl. Math. 21, 631-652 (1968)

6. Hayakawa, K.: On nonexistence of global solutions of some semilinear parabolic differential equations. Proc. Jpn. Acad. 49, 503-505 (1973)

7. Hayashi, N., Kaikina, E.I., Naumkin, P.I.: Asymptotics for fractional nonlinear heat equations. J. Lond. Math. Soc. 72, 663-688 (2005)

8. Ishige, K., Kawakami, T., Kobayashi, K.: Asymptotics for a nonlinear integral equation with a generalized heat kernel. J. Evol. Equ. 14, 749-777 (2014)

9. Kobayashi, K., Sirao, T., Tanaka, H.: On the growing up problem for semilinear heat equations. J. Math. Soc. Jpn. 29(3), 407-425 (1977)

10. Laister, R., Robinson, J.C., Sierżęga, M., Vidal-López, A.: A complete characterisation of local existence for semilinear heat equations in Lebesgue spaces. Ann. Inst. H. Poincaré Anal. Non Linéaire 33(6), 1519-1538 (2016)

11. Li, K.: A characteristic of local existence for nonlinear fractional heat equations in Lebesgue spaces. Comput. Math. Appl. 73, 653-665 (2017)

12. Meneses, R., Quaas, A.: Fujita type exponent for fully nonlinear parabolic equations and existence results. J. Math. Anal. Appl. 376, 514-527 (2011)

13. Miao, C., Yuan, B., Zhang, B.: Well-posedness of the Cauchy problem for the fractional power dissipative equations. Nonlinear Anal. 68, 461-484 (2008)

14. Nagasawa, M., Sirao, T.: Probabilistic treatment of the blowing up of solutions for a nonlinear integral equation. Trans. Am. Math. Soc. 139, 301-310 (1969)

15. Quittner, P., Souplet, Ph: Superlinear Parabolic Problems. Blow-up, Global Existence and Steady States. Birkhäuser Advanced Texts, 2nd edn. Birkhäuser, Basel (2019)

16. Robinson, J.C., Sierżęga, M.: Supersolutions for a class of semilinear heat equations. Rev. Mat. Complut. 26, 341-360 (2013)

17. Sugitani, S.: On nonexistence of global solutions for some nonlinear integral equations. Osaka J. Math. 12, 45-51 (1975)

Publisher's Note Springer Nature remains neutral with regard to jurisdictional claims in published maps and institutional affiliations. 